\title{
Post-Stroke Depression or Fatigue?
}

\author{
Fabienne Staub Julien Bogousslavsky \\ Department of Neurology, Centre Hospitalier Universitaire Vaudois, Lausanne, Switzerland
}

Fatigue is a common complaint in clinical practice. It is a frequent symptom in patients with depression and other psychiatric disorders, cancer, inflammatory, endocrinologic and rheumatologic diseases. It may also develop without any other antecedent condition than a viral infection, leading to what is generally known as the 'chronic fatigue syndrome'. In neurology, most studies have been performed in multiple sclerosis, but there are some investigations on fatigue in Parkinson's disease, postpolio syndrome, immune-mediated polyneuropathies, systemic lupus erythematosus, Lyme disease and amyotrophic lateral sclerosis.

Although fatigue is a frequent, often disabling, poststroke sequela, there have been only a few investigations on the subject [1-3]. Furthermore, fatigue was usually addressed just as one symptom of poststroke depression. This is why the study by van der Werf et al. in the present issue of European Neurology is particularly timely.

The first issue to resolve is a definition problem. Indeed, although there is a popular knowledge of fatigue, the vagueness which surrounds the concept makes difficult any operational definition.

The first and most obvious distinction is between objective and subjective fatigue. Objective fatigue is the observable and measurable decrement in performance occurring with the repetition of a physical or mental task, while subjective fatigue is a feeling of early exhaustion, weariness and aversion to effort. In their paper, van der Werf et al. have chosen the term of 'experienced fatigue' to describe this feeling of fatigue, which is indeed most relevant to clinical studies.
It can also be useful to distinguish between fatigue which develops in connection with activities requiring a sustained effort (fatigability), and fatigue as a primary state which is closer to a lack of initiative with imbalance between motivation (preserved) and effectiveness (decreased).

It is also possible to divide the concept of fatigue into various subtypes, namely 'physical' fatigue occurring after muscular exertion, 'somatic' fatigue related to disease, 'mental' fatigue (for example appearing with cognitively demanding tasks or in the presence of neuropsychological disorders) and 'psychological' fatigue (for example associated with lack of interest or poor motivation). These subtypes of fatigue (which are not mutually exclusive) can then be expressed at a behavioral level (objective fatigue) or as a state of feeling (subjective fatigue).

The limit between normal and pathological fatigue is not clear. The duration ('chronicity') and severity of fatigue, associated disability (interference with daily functioning), other symptoms and the request for a medical consultation are signs which point towards an abnormal situation. Van der Werf et al. found that $51 \%$ of their stroke outpatients experienced severe fatigue against only $16 \%$ of the controls. This difference was also found by Ingles et al. [1], but with a higher proportion of selfreported fatigue problems (68\% vs. $36 \%)$. In our own experience, poststroke fatigue is often quantitatively and qualitatively different from premorbid fatigue, which is in agreement with studies on fatigue in Parkinson's disease [4] and multiple sclerosis [5].

\begin{tabular}{ll}
\hline KARGER & ( ) 2001 S. Karger AG, Basel \\
0014-3022/01/0451-0003\$17.50/0 \\
$\begin{array}{l}\text { Fax +4161306 12 34 } \\
\begin{array}{l}\text { E-Mail karger@karger.ch } \\
\text { www.karger.com }\end{array}\end{array}$ & $\begin{array}{l}\text { Accessible online at: } \\
\text { www.karger.com/journals/ene }\end{array}$
\end{tabular}

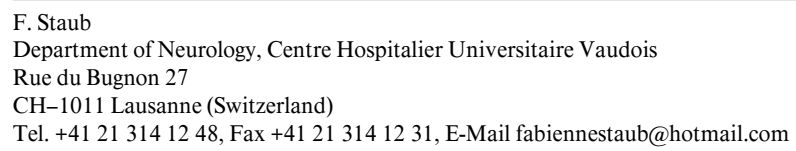


A feeling of fatigue is often described using related terms, such as asthenia, apathy, weariness, lassitude, abulia, exhaustion, boredom or lack of energy. This diversity illustrates well the variety of phenomena which are commonly included, sometimes inappropriately, under the term of fatigue. It is thus essential to clarify the semantic field of subjective fatigue and to distinguish it from distinct, although close, phenomena, such as depression and abulia. The overlap between fatigue and depression is undeniable, and the first indeed constitutes one of the criteria for depression in most scales. Nevertheless, fatigue can occur in the absence of depression, a dissociation which has been highlighted in Parkinson's disease [4] and multiple sclerosis $[5,6]$, and which has also been found in stroke patients [1]. Van der Werf et al. found that only $38 \%$ of patients with severe fatigue were also depressed. Ingles et al. [1] reported similar findings, with $29 \%$ of patients having both symptoms.

To date, the study of poststroke fatigue has been nearly completely neglected, and there are many questions which have not been solved, or even asked.

It is for example necessary to delineate the characteristics (pre-, per- and poststroke) associated with the development of fatigue. Van der Werf et al. found no association between fatigue and demographic data (age, gender) and stroke-related features (lesion side and site, delay poststroke), but they found a positive relation between fatigue and functional disability, mainly for locomotion impairment. In the study by Ingles et al. [1], neither stroke-related features (type of infarct, lesion location, lesion lateralization) nor neurologic and functional impairment seemed to play a role. It would be particularly interesting to assess the influence of cognitive deficits on fatigue, as well as the role of premorbid psychological factors, which may be critical for the development of poststroke depression [7].

A second direction of research is to study the nature of poststroke fatigue. Is there one or several types of poststroke fatigue? Subjective fatigue is a multidimensional state, which includes sensory, emotional and cognitive components. That does not say whether the feeling of fatigue refers to a primary or a secondary feeling state. Intuitively, one can easily admit that a neuropsychological deficit generates mental fatigue, and neurologic impairment physical fatigue. The psychological component of poststroke fatigue must also be taken into account. According to the coping hypothesis, fatigue is due to a compensatory effort to meet the demands of normal life following disease. Poststroke sleep disturbances have also to be considered, since they may cause fatigue with day somnolence. Once relationships between fatigue and stroke sequelae, psychological factors and sleep disorders are determined, the question of a 'primary' poststroke fatigue (i.e. without coexisting depression, significant cognitive impairment or any other clinical dysfunction which may cause fatigue) can be addressed. In a pilot study conducted in our cerebrovascular neurobehavioral clinic [8], we found a high proportion of brainstem and thalamic lesions among fatigued patients without significant stroke sequelae or mood disorder. That led us to think that primary poststroke fatigue exists and may be linked to minor attentional deficits due to the interruption of neural networks, such as the reticular activating system. In their paper, van der Werf et al. mention the influence of neurologic and neuropsychological impairment, mood disorders, sleep disturbances and coping factors in the genesis of fatigue. Their study shows a clear contribution of physical impairment and depression (less important), but the link between neuropsychological sequelae and fatigue seems weak. In fact, the quantification of cognitive impairment was only based on self-report, not on formal testing, which may explain this finding. We are convinced that neuropsychological deficits, in particular executive and aphasic, do play a role in the genesis of (mental) fatigue. However, it is striking to note that patients with very good recovery are often extremely disabled by fatigue, which may even prevent them to resume social and professional activities. In a study on mental fatigue in patients with supratentorial lacunar infarct, Van Zandvoort et al. [2] found that despite very good neurologic recovery and normal cognitive abilities, the patients showed a decrement of performances (compared to controls) in demanding tasks, which seemed to be linked to subtle attentional deficits.

Thus, the causes leading to a feeling of fatigue after stroke may be varied. Whether this state of feeling is identical whatever the underlying condition is an open question. The development of an evaluative instrument is not an easy matter, first because the feeling of fatigue is a multidimensional state, second because it is poorly correlated with objective measures and therefore must rely mostly on the psychological attributes of the experience. A variety of self-reported instruments have been developed to measure fatigue as a feeling state. Some have been created for specific disorders [9, 10], others are more globally applicable, being often included in general health inquiry. Some scales test general fatigue [11], others mental [12] or physical fatigue, while others are multidimensional and assess cognitive, physical and psychological components of fatigue $[9,10]$. The fatigue subscale Checklist Individu- 
al Strength which was used in the study by van der Werf et al. is an instrument which essentially assesses the physical component of fatigue. This may explain why the authors found such a strong relationships between fatigue score on this scale and physical impairment. We believe that a multidimensional instrument is necessary to assess the complexity of subjective fatigue.
Fatigue is a frequent and potentially disabling poststroke sequela, which does not seem to always depend on depression. Further longitudinal prospective studies are needed to understand the pre-, per- and poststroke characteristics which are associated with the development of fatigue, as well as its relationships with depression, poststroke neurologic and neuropsychological sequelae and psychological factors.

\section{References}

1 Ingles JL, Eskes GA, Phillips SJ: Fatigue after stroke. Arch Phys Med Rehabil 1999;80:173178.

2 Van Zandvoort MJE, Kappelle LJ, Algra A, et al: Decreased capacity for mental effort after single supratentorial lacunar infarct may affect performance in everyday life. J Neurol Neurosurg Psychiatry 1998;65:697-702.

3 Leegaard OF: Diffuse cerebral symptoms in convalescents from cerebral infarction and myocardial infarction. Acta Neurol Scand 1983;67:348-355.

4 Friedman J, Friedman H: Fatigue in Parkinson's disease. Neurology 1993;43:2016-2019.
5 Vercoulen J, Hommes OR, Swanink C, et al: The measurement of fatigue in patients with multiple sclerosis: A multidimensional comparison with patients with chronic fatigue syndrome and healthy subjects. Arch Neurol 1996; 53:642-649.

6 Van der Werf SP, Jongen PJ, Lycklama a Nijeholt GJ, et al: Fatigue in multiple sclerosis: Interrelations between fatigue complaints, cerebral MRI abnormalities and neurological disability. J Neurol Sci 1998;160:164-170.

7 Pohjasvaara T, Leppavuori A, Siira I, et al: Frequency and clinical determinants of poststroke depression. Stroke 1998;29:2311-2317.

8 Staub F, Annoni JM, Bogousslavsky J: Fatigue after stroke: A pilot study. Cerebrovasc Dis 2000;10(suppl 2):62.
9 Fisk JD, Ritvo PG, Ross L. et al: Measuring the functional impact of fatigue: Initial validation of the fatigue impact scale. Clin Infect Dis 1994;18(suppl 1):79-83.

10 Schwartz JE, Jandorf L, Krupp LB: The measurement of fatigue: A new instrument. J Psychosom Res 1993;37(7):753-762.

11 Lee KA, Hicks G, Nino-Murcia G: Validity and reliability of a scale to assess fatigue. Psychiatry Res 1991;36:291-298.

12 Bentall RP, Wood GC, Marrinan T, et al: A brief mental fatigue questionnaire. Br J Clin Psychol 1993;32:375-379. 